\title{
Article \\ Filtered Influence Function of Deformable Mirror for Wavefront Correction in Laser Systems
}

\author{
Yamin Zheng ${ }^{1,2} \mathbb{D}^{\mathbb{D}}$, Ming Lei ${ }^{3}$, Shibing Lin ${ }^{1,2}$, Deen Wang ${ }^{1,2,4}$, Qiao Xue ${ }^{4}$ and Lei Huang ${ }^{1,2, *}$ \\ 1 Key Laboratory of Photonic Control Technology Ministry of Education, Department of Precision Instrument, \\ Tsinghua University, Beijing 100084, China; yamin.zheng@foxmail.com (Y.Z.); \\ linsb19@mails.tsinghua.edu.cn (S.L.); sduwde@126.com (D.W.) \\ 2 State Key Laboratory of Precision Measurement Technology and Instruments, Department of Precision \\ Instrument, Tsinghua University, Beijing 100084, China \\ 3 China Defense Science and Technology Information Center, Beijing 100084, China; minglei_cdstic@163.com \\ 4 Research Center of Laser Fusion, China Academy of Engineering Physics, P.O. Box 919-988, \\ Mianyang 621900, China; 031205100@163.com \\ * Correspondence: hl@tsinghua.edu.cn
}

check for

updates

Citation: Zheng, Y.; Lei, M.; Lin, S.; Wang, D.; Xue, Q.; Huang, L. Filtered Influence Function of Deformable Mirror for Wavefront Correction in Laser Systems. Photonics 2021, 8, 410. https: / / doi.org/10.3390/ photonics 8100410

Received: 20 August 2021

Accepted: 16 September 2021

Published: 23 September 2021

Publisher's Note: MDPI stays neutral with regard to jurisdictional claims in published maps and institutional affiliations.

Copyright: (c) 2021 by the authors. Licensee MDPI, Basel, Switzerland. This article is an open access article distributed under the terms and conditions of the Creative Commons Attribution (CC BY) license (https:/ / creativecommons.org/licenses/by/ $4.0 /)$.

\begin{abstract}
An influence function filtering method (IFFM) is presented to improve the wavefront correction capability in laser systems by curbing the correction performance degradation resulted from the IF measurement noise. The IFFM is applied to the original measured IF. The resulting filtered IF is then used to calculate the wavefront control signal in each iteration of the closed-loop correction. A theoretical wavefront correction analysis model (CAM) is built. The impact of the IF measurement noise as well as the improvement of the IFFM on the wavefront correction capability are analyzed. A simulation is set up to analyze the wavefront correction capability of the filtered IF using Zernike mode aberrations. An experiment is carried out to study the effectiveness of the IFFM under practical conditions. Simulation and experimental results indicate that the IFFM could effectively reduce the negative effect of the measurement noise and improve the wavefront correction capability in laser systems. The IFFM requires no additional hardware and does not affect the correction speed.
\end{abstract}

Keywords: adaptive optics; influence function; wavefront correction

\section{Introduction}

Adaptive optics (AO) is widely employed in many fields, from astronomical observation [1-8] at the macro level to bioimaging microscopy [9-15] at the micro level and from inertial confinement fusion facilities [12-14] at the high-power level to vision science research [15-17] at the low-power level. One of the important applications of AO is wavefront correction in laser systems to improve the beam quality of the output laser beam [18-20]. An AO system generally consists of a wavefront sensor, a wavefront controller and a wavefront compensator. The deformable mirror (DM) is commonly adopted as the wavefront compensator [21,22]. In the wavefront correction process, the DM's influence function (IF) and the distorted wavefront to be corrected are measured by the wavefront sensor, and then the measured data are transmitted to the wavefront controller to calculate the control signal of the DM. The surface shape of the DM is then deformed to generate the conjugated wavefront to correct the wavefront aberration. Therefore, the measurement accuracy of the IF should be high enough to ensure the accuracy of the calculated control signal so as to obtain fine correction capability. However, in many practical optical systems, the IF is commonly measured by using a Shack-Hartmann wavefront sensor (SHWFS) and the measurement noises always exists in the measured IF [23-25]. According to previous research, the measurement noises occur in the form of random and irregular fluctuations with amplitude being about $30 \%$ of the overall deformation amplitude of the DM caused by ambient vibration and turbulence [23-25]. Due to occurrence of the measurement noises, 
the measured IF is inconsistent with the actual IF of the DM, and the wavefront correction ability of the AO system is limited.

Some studies have been focused on the improvement of the measurement accuracy of the IF to obtain good wavefront correction capability [26-29]. An adaptive-influence-matrix (AIM) method was proposed, in which the IF was measured and calibrated multiple times during the closed-loop correction process to improve the measurement accuracy [26]. This method took the nonlinearity effect of the actuators into consideration while leaving out the random and irregular measurement noise. Then, a two-step high-precision system identification method in wavefront control was proposed [27] with the measurement noise being considered, in which multiple tentative measured wavefronts were used to suppress the measurement noise and obtain optimized influence functions. Besides, a wavefront reconstruction method was proposed [28] to estimate the high-resolution wavefront using multiple wavefront measurements. The high-resolution wavefront was reconstructed by a maximum a posteriori (MAP) method, taking into consideration the measurement model and the prior information derived from the spectrum statistics of the turbulence phase. The accuracy of the measured IF could hence be improved. Nonetheless, in these methods that could improve the IF measurement accuracy, multiple IF measurements were required, which would reduce the applicability of the AO system. Recently, a hybrid AO systembased IF optimization method was proposed [29], in which the IF was measured by using a deflectometry system with higher measurement accuracy than the SHWFS. Simulation and experimental results revealed that the method could improve the IF measurement accuracy as well as the wavefront correction capability, however the utilization of additional hardware would increase the structure complexity of the system. In addition to this, some studies focused on fitting the actual IF with Gaussian function and its modifications [30,31]. A modified Gaussian influence function (MGIF) was proposed to fit the IF measured by a Zygo interferometer [30], and a simple method is adopted to characterize the actual IF of various position actuators. Compared to the traditional Gaussian IF, the MGIF possesses the actual IF features in the azimuthal and radial directions. Besides, the IF of a 140 -actuator continuous membrane DM was measured using an SHWFS and then fitted using a Gaussian function [31]. The Gaussian and modified Gaussian function fitting methods could be implemented in the IF measurement during the precorrection process, which means that the effect of the measurement noise could be mitigated in the correction; therefore, the closed-loop correction capability could be improved. Nonetheless, this requires that the IF needs to be accurately measured in the precorrection process without the measurement noise, thus reducing the flexibility of the measurement.

An IF filtering method (IFFM) to effectively improve the closed-loop wavefront correction capability of the AO system in laser systems is presented in this paper. The IFFM is applied during the calculation process of the wavefront correction. In the IFFM, a filtered IF is generated in the precorrection process based on the measured IF through the filtering function. After that, the filtered IF will replace the measured IF in the closed-loop correction process to calculate the wavefront correction control signal with the measured laser wavefront. The filtered-IF-based control signal is then applied to the DM to improve the closed-loop correction capability of the AO system. This paper is organized as follows. In Section 2, we build a theoretical wavefront correction analysis model (CAM) in simulation to systematically analyze the impact of the measurement noises on the correction capability. We also introduce principles of the IFFM, including the calculation process and the characteristic analysis of the IF before and after the filtering. In Section 3, a simulation model is built to investigate the improvement of correction capability brought by the IFFM. In Section 4, an experiment is conducted to study the effectiveness of the IFFM in practical circumstances. In Section 5, we analyze and discuss the simulation and experimental results, which show that the correction capability as well as the robustness of the AO system could be improved by using the IFFM. 


\section{Principles}

\subsection{Principles of the Wavefront Correction Analysis Model}

In an AO system, in which a DM is employed as the wavefront compensator and the IF of the DM is taken to calculate the control signals, the measurement noise of the IF has a negative effect on the correction capability of the system. To theoretically investigate the effect of measurement noises, a theoretical CAM is built. This model explains how IF works in the wavefront correction process of an $\mathrm{AO}$ system, and clarifies the relationship of the IF, the initial wavefront (which is to be corrected) and the residual wavefront after correction. Symbols used in the CAM and corresponding descriptions are listed in Table 1.

Table 1. Symbols used in the CAM.

\begin{tabular}{cc}
\hline Symbol & Description \\
\hline$w$ & Initial wavefront before correction \\
$r$ & Residual wavefront after correction \\
$u$ & Control signal of the DM \\
$F_{a}$ & Simulated ideal IF of the DM \\
$F_{\text {measu }}$ & Measured IF of the DM \\
$F_{\text {modu }}$ & Filtered IF of the DM \\
$\delta$ & Measurement noise of the IF \\
\hline
\end{tabular}

The parameters in the CAM are defined as follows. The initial wavefront $w$ represents the wavefront measured by the wavefront sensor and to be corrected by the AO system. The control signal $u$ represents the voltage signal that is applied on the DM to generate conjugate wavefront to compensate the initial wavefront $w$. The residual wavefront $r$ represents the uncorrected residual after the correction of the initial wavefront $w$. The ideal IF $F_{a}$ represents the IF simulated based on the model. The measured IF $F_{\text {measu }}$ represents the DM's IF measured by the wavefront sensor. The filtered IF $F_{m o d u}$ represents the IF filtered from the measured IF $F_{\text {measu }}$ by the IFFM. The IF measurement noise $\delta$ represents the difference between the measured IF $F_{\text {measu }}$ and the ideal IF $F_{a}$.

In the CAM, the wavefront correction process of the AO system is simplified into two steps. In step one, $w$ is set as the compensation target and $u$ is calculated using $F_{c}$ and $w$ through a calculation algorithm. Here, we adopt the least squares method, a form of mathematical regression analysis often used in $\mathrm{AO}$ wavefront correction, to calculate the control signal $u$ that best fits the DM's IF and the wavefront aberration to be corrected [32-34]. In step two, the compensating wavefront is generated using the ideal IF $F_{a}$ and the control signal $u$, and the residual $r$ is calculated as the difference between $w$ and the compensating wavefront. The two steps are expressed as Equations (1) and (2):

$$
\begin{gathered}
F_{c} u=-w, \\
r=w+F_{a} u,
\end{gathered}
$$

where $F_{c}$ represents the IF (i.e., $F_{a}, F_{\text {measu }}$ or $F_{\text {modu }}$ ) that is used in the calculation of the control signal $u$. Taking Equation (2) as a linear system of equations, there exists an optimal control signal $u_{o p}$ that could minimize the residual, and $u_{o p}$ is given by

$$
u_{o p}=-\left(F_{a}^{T} F_{a}\right)^{-1} F_{a}^{T} w .
$$

Meanwhile, the solution $u_{c}$ of $u$ in Equation (1) is given by

$$
u_{c}=-\left(F_{c}^{T} F_{c}\right)^{-1} F_{c}^{T} w .
$$


It is noteworthy that $F_{a}$ in Equation (2) represents the ideal IF and could not be accurately measured in experiments since measurement noise $\delta$ always exists. Thus, it could be seen that $u_{o p}$ and $u_{c}$ are not identical invariably, which will finally lead to the differences in wavefront correction capability.

$F_{\text {measu }}$ is used as $F_{c}$ in practical correction process:

$$
F_{c}=F_{\text {measu }}=F_{a}+\delta \text {. }
$$

If the measurement is ideal and no measurement noise (i.e., $\delta=0$ ) exists, $F_{c}$ and $u_{c}$ equal the ideal IF $F_{a}$ and $u_{o p}$, respectively, and the optimal $r$ could be obtained. However, the ideal measurement could not be achieved practically due to ambient vibration and turbulence. The measurement noise $\delta$ does not equal zero and $F_{c}$ equals the measured IF $F_{\text {measu }}$, while $u_{c}$ is not equal to $u_{o p}$. Thus, the wavefront correction capability is affected.

Previous research shows that for an AO system operating indoors, the IF measurement noise $\delta$ appears as random and irregular fluctuations in the whole clear aperture [18-20]. For each actuator's IF, the peak-to-valley (PV) value of the measured noise $\delta$ is generally $20-40 \%$ of that of the ideal IF $F_{a}$. Due to the measurement noise, the optimal $r$ could not be obtained in a practical AO system, which will result in ability degradation of the wavefront correction.

A simulation model is built to investigate the effect of the measurement noise $\delta$ on the residual $r$. In the simulation, the ideal IF $F_{a}$ is built through the COMSOL Multiphysics software, while the measured IF $F_{\text {measu }}$ as well as the correction process are simulated through MATLAB software [35,36].

As shown in Figure 1, a finite element model of the DM, which consists of a base, a mirror and 49 in-between actuators, is built in COMSOL. Structural and material parameters of the DM are listed in Tables 2 and 3 separately. BK7, stainless steel and piezoelectric ceramics are set as materials of the mirror, the base and the actuators, respectively. The model is based on the stress interface in the structural mechanics module. The vertical deformation, horizontal rotation and center point of the base undersurface are all set to zero degrees of freedom. The initial surface shape of the mirror is set in an absolute plane. In the simulation, the top faces of the 49 actuators are set to shift up by $1 \mu \mathrm{m}$ individually in order to obtain the ideal IF $F_{a}$, and the resulting mirror surface shape deformations are recorded as the IFs in forms of matrices accordingly.

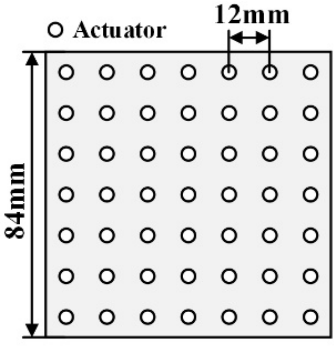

(a)

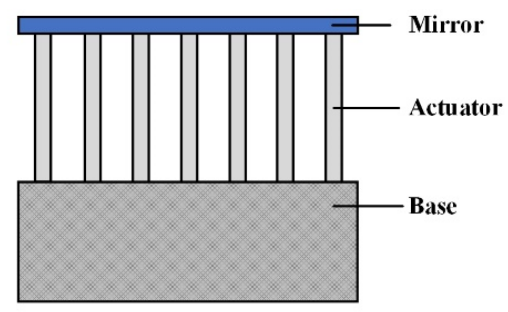

(b)

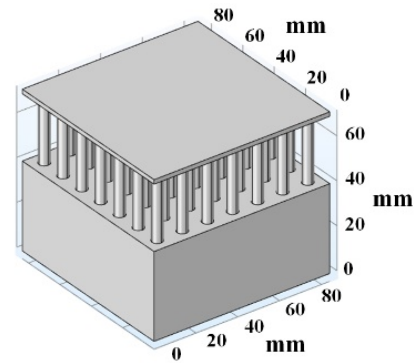

(c)

Figure 1. Schematic diagrams of the DM. (a) Actuator distribution, (b) side view of structure and (c) COMSOL model of the DM.

Table 2. Structural parameters of the DM.

\begin{tabular}{cccc}
\hline Parameter & Mirror & Actuator & Base \\
\hline Value $/ \mathrm{mm}$ & $84 \times 84 \times 2$ & $\Phi 5 \times 30$ & $84 \times 84 \times 40$ \\
\hline
\end{tabular}


Table 3. Material parameters of the DM.

\begin{tabular}{cccc}
\hline Parameter & BK7 & Stainless Steel & Piezoelectric Ceramics \\
\hline Young's Modulus $/ \mathrm{GPa}$ & 81 & 193 & 75 \\
Poisson's Ratio & 0.17 & 0.3 & 0.35 \\
Density $/ \mathrm{kg} \cdot \mathrm{m}^{-3}$ & 2400 & 7930 & 7750 \\
Linear Expansivity $/ 10^{-6} \cdot \mathrm{K}^{-1}$ & 7.1 & 17.2 & 12 \\
\hline
\end{tabular}

In the simulation, in order to calculate the measured IF $F_{\text {measu }}$, the IF measurement noise $\delta$ is generated and added to the ideal IF $F_{a}$ in MATLAB software. The measurement noise $\delta$ used in the simulation is based on the noise data measured under experimental conditions. The experimental noise data is measured on an optical table equipped with four self-leveling active isolation table legs to avoid strong vibrations, and the fans of the cleanroom are closed to avoid strong air turbulence. The experimentally measured noise data have the characteristics of random distribution as well as low amplitude. Therefore, the noise $\delta$ for each actuator is set as continuous and irregular in the whole clear aperture, while the PV value of the noise is set $30 \%$ of that of the ideal IF $F_{a}$. Thus, for each actuator, the noise $\delta(i)$ is added to the ideal IF $F_{a}(i)$ ( $i$ from 1 to 49 ) and the measured IF $F_{\text {measu }}(i)$ is obtained as

$$
F_{\text {measu }}(i)=F_{a}(i)+\delta(i), \quad i=1,2, \ldots, 49 .
$$

For all the 49 actuators, the measurement noises are set random and distinct:

$$
\delta(i) \neq \delta(j), \quad i, j=1,2, \ldots, 49, \quad i \neq j .
$$

Taking the 25th actuator (i.e., the central actuator of the DM) as an example, Figure 2 illustrates its simulated ideal IF $F_{a}(25)$, measurement noise $\delta(25)$ and measured IF $F_{\text {measu }}(25)$. It could be seen that in the simulated ideal IF $F_{a}(25)$, the surface fluctuation is concentrated in the form of a peak and the height gradually decreases outwards from the center. After the measurement noise $\delta(25)$ is added to $F_{a}(25)$, the simulated measured IF $F_{\text {measu }}(25)$ is obtained with random fluctuations in the whole clear aperture.

$F_{a}(25)$

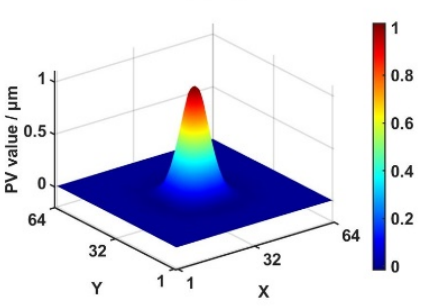

(a)

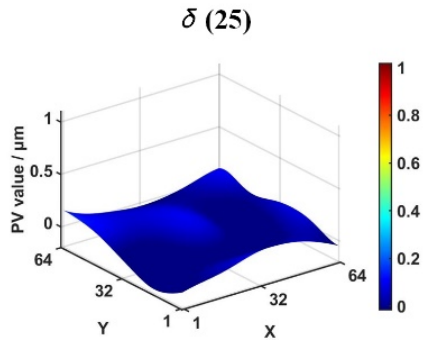

(b)

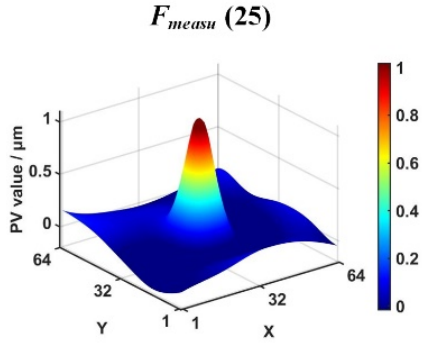

(c)

Figure 2. Comparison between $F_{\text {measu }}$ and $F_{a}$, taking the 25th actuator as example. (a) $F_{a}(25)$, (b) $\delta(25)$ that is added to the $F_{a}(25)$, (c) $F_{\text {measu }}(25)$.

The Gaussian function has been employed to fit the measured IF in many studies with the expression of

$$
G(x, y)=A \cdot \exp \left[\ln (\omega) \cdot \frac{\left(x-c_{x}\right)^{2}+\left(y-c_{y}\right)^{2}}{2 \sigma^{2}}\right]
$$

where $A$ is the peak amplitude, $\omega$ is the coupling coefficient, $c_{x}$ and $c_{y}$ are the coordinates of the Gaussian function center, and $\sigma$ is the standard deviation value $[30,31]$. The generated ideal IF $F_{a}(25)$ could be fitted by the Gaussian function with the parameters set $A=0.9841$, $\omega=0.15$ and $\sigma=8$. The root-mean-square (RMS) value of the fitting residual is $0.0087 \mu \mathrm{m}$, which is $93 \%$ smaller than the RMS value of $F_{a}(25)$ (i.e., $0.1208 \mu \mathrm{m}$ ). 
The wavefront correction abilities of the DM using the ideal IF $F_{a}$ and the measured IF $F_{\text {measu }}$ are verified by taking the 3 rd to 10 th Zernike mode aberrations $[37,38]$ as the initial wavefronts. In step one of the correction process, $F_{a}$ and $F_{\text {measu }}$ are set as $F_{\mathcal{c}}$ (Equation (1)), respectively. In step two, $F_{a}$ is used to calculate the residual (Equation (2)). PV values and RMS values of the corrected wavefront are depicted in Figure $3 a, b$, separately. In Figure 3 , the blue bars represent the correction results when no measurement noise occurs and $F_{c}$ equals the ideal IF $F_{a}$, while orange bars represent the results when measurement noise exists and $F_{c}$ equals the measured IF $F_{\text {measu }}$. As shown in Figure 3, the PV values of the corrected wavefront aberrations by using $F_{a}$ is $31 \%$ lower on average than those by using $F_{\text {measu }}$. For the RMS values, the reduction is as large as $47 \%$. This indicates that the measured IF $F_{\text {measu }}$ is less effective than the ideal IF $F_{a}$ in the wavefront correction for the 3rd to 10th Zernike mode aberrations.

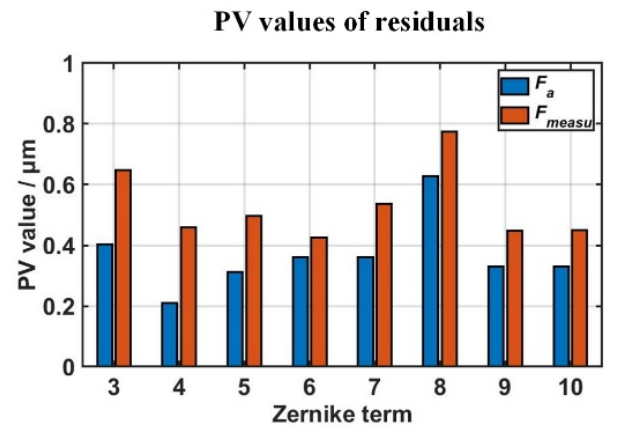

(a)

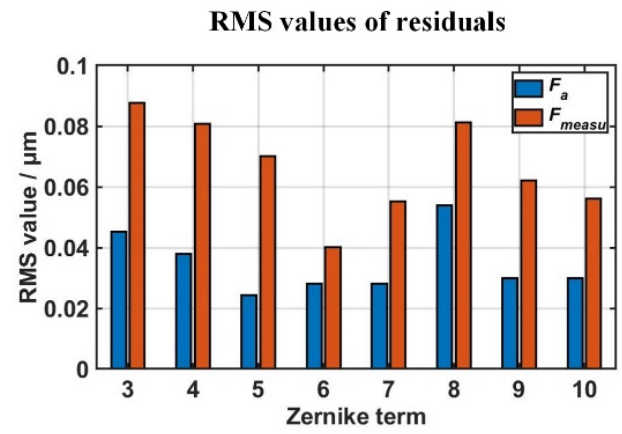

(b)

Figure 3. (a) PV and (b) RMS values of correction residuals of the 3rd-10th Zernike mode aberrations. The blue bars represent the correction results by using $F_{a}$, and the orange bars by using $F_{\text {measu }}$.

In conclusion, simulation results suggest that $F_{a}$ has better correction capability than $F_{\text {measu }}$. In other words, the measurement noise $\delta$ would result in the degradation of the wavefront correction ability. To reduce the negative effect of the measurement noise $\delta$ on the AO system's capability, the IFFM is proposed.

\subsection{Principles of IFFM}

In the wavefront correction process of an $\mathrm{AO}$ system, the DM operates as the wavefront compensator while the measured IF ( $\left.F_{\text {measu }}\right)$ of the DM is used along with the initial wavefront $w$ to calculate the control signal $u$. The IFFM is introduced into step one of the CAM (i.e., the calculation process of the control signal) to restrain the degradation of the wavefront correction capability brought by the measurement noise $\delta$. The IFFM filters the measured IF $F_{\text {measu }}$ into the filtered IF $F_{\text {modu }}$, which is used for the calculation of the control signal.

Filtering methods used in the IFFM should abide by two principles: the characteristics of central peak of the measured IF $F_{\text {measu }}$ in certain areas around the center should be preserved, and the random noise of the measured IF $F_{\text {measu }}$ in the area far from the center should be suppressed. Based on the two principles, three types of filtering methods are proposed, including the cut-type filtering, the linear-type filtering and the Gaussian-type filtering, to reduce the measurement noise $\delta$ without losing the characteristics of the central peak. The filtered IF $F_{m o d u}$ is given by

$$
F_{\text {modu }}(i)=M(i) \odot F_{\text {measu }}(i) i=1,2, \ldots, 49 .
$$

Here, the filtering matrix $M(i)\left[M_{1}(i), M_{2}(i)\right.$ and $\left.M_{3}(i)\right]$ is used to filter the measured IF $F_{\text {measu }}(i)$. The symbol $\odot$ means the element-wise product of matrices, where each element $j, k$ of the new matrix is the product of elements $j, k$ of the original two matrices. The $F_{\text {modu }}(i)$ matrix is the element-wise product of the $F_{\text {measu }}(i)$ matrix and the $M(i)$ matrix. To compare the filtering matrices, the central actuator of the DM (i.e., the 25th actuator) 
is taken as an example. Shown in Figure 4 are the element values of $M_{1}(25), M_{2}(25)$ and $M_{3}(25)$. The filtering matrices cover the range of the full DM's surface with a size of 64 by 64 pixels.

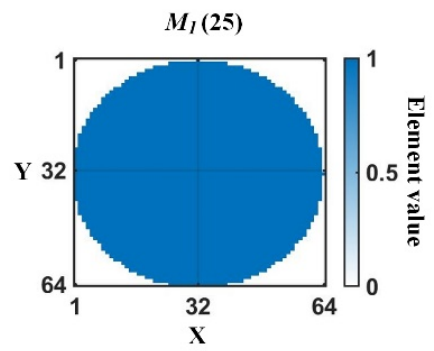

(a)

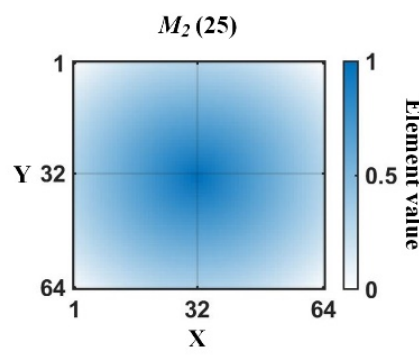

(b)

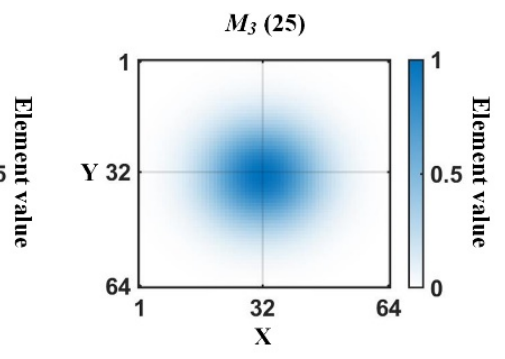

(c)

Figure 4. Values of the elements of (a) $M_{1}$ (25) for cut-type filtering, (b) $M_{2}$ (25) for linear-type filtering and (c) $M_{3}(25)$ for Gaussian-type filtering.

The first type is the cut-type filtering. For the filtering matrix $M_{1}(i)$ of each actuator, a circular area is specified and the elements inside it have the same value of 1 , while the elements outside it have the same value of 0 . The position of the element with the largest value of $F_{\text {measu }}(i)$ is selected as the center of the circle area of $M_{1}(i)$. Then, the position of the nearest element with the value being reduced by $95 \%$ or more of $F_{\text {measu }}(i)$ is selected, and the distance between the selected position and the circle's center is taken as the radius of the circular area of $M_{1}(i)$. It could be seen in Figure 4 a that the cut-type filtering could preserve the characteristics in the circular area near the peak of the measured IF $F_{m e a s u}$, and the characteristics outside the circular area are eliminated.

The second type is the linear-type filtering. For the filtering matrix $M_{2}(i)$ of each actuator, the elements' values are considered decreasing linearly from 1 to 0 outwards from the specified center. Like $M_{1}(i)$, the position of the element with the largest value of $F_{\text {measu }}(i)$ is selected as the center, and the element of $M_{2}(i)$ at this position has the value of 1. The element of $M_{2}(i)$ with the furthest distance from the specified center has the value of 0 . The value of each element of $M_{2}(i)$ depends on the element's distance from the specified center and decreases linearly with the distance. It could be seen in Figure $4 \mathrm{~b}$ that the lineartype filtering modulates the whole aperture of the measured IF $F_{\text {measu }}$. Compared with other two types, the linear-type filtering preserves all the characteristics of the measured IF $F_{\text {measu }}(i)$, including the central peak characteristics (i.e., the ideal IF $F_{a}$ ) and the random fluctuations (i.e., the measurement noise $\delta$ ).

The third type is the Gaussian-type filtering. For the filtering matrix $M_{3}(i)$ of each actuator, elements' values are considered decreasing nonlinearly with distance from the specified center and conforming to the Gaussian distribution. Like $M_{1}(i)$ and $M_{2}(i)$, the position of the element with the largest value of $F_{\text {measu }}(i)$ is selected as the center, and the element of $M_{3}(i)$ at the same position has the value of 1 . The position of the nearest element with the value reduced by $95 \%$ or more of $F_{\text {measu }}(i)$ is selected, and the distance between the selected position and the specified center is taken as the standard deviation $\sigma$ of the Gaussian distribution. It could be seen from Figure $4 \mathrm{c}$ that the Gaussian-type filtering modulates the whole aperture and partially preserved the fluctuations of the measured IF $F_{\text {measu }}$. However, compared with those in the central area, the fluctuations outside are greatly suppressed.

Filtering results of the measured IF by using these three types of filtering methods are depicted in Figure 5, taking the 25th actuator as an example. Illustrated in Figure 6 are the cutaway views of the surface shape deformations of the IFs. 


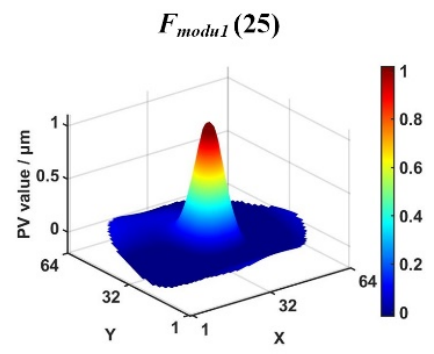

(a)

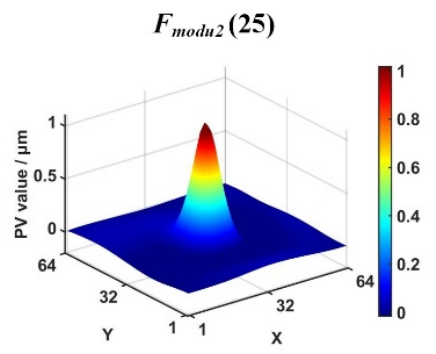

(b)

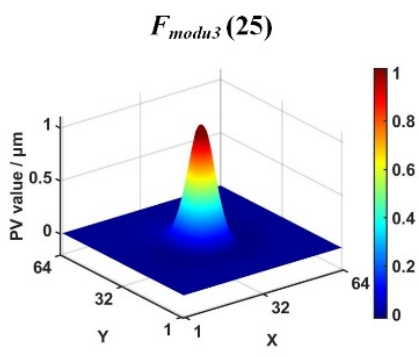

(c)

Figure 5. Filtered IFs. (a) Cut-type filtering result $F_{\text {modu1 }}(25)$. (b) Linear-type filtering result $F_{\text {modu2 }}(25)$. (c) Gaussian-type filtering result $F_{\text {modu } 3}(25)$.

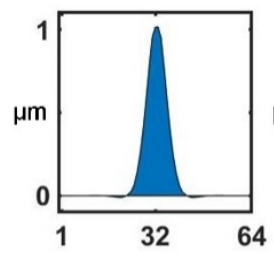

(a)

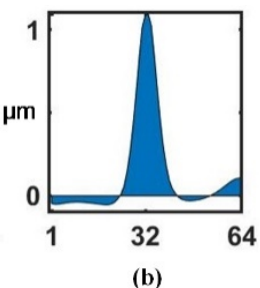

(b)

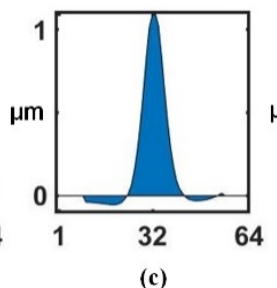

(c)
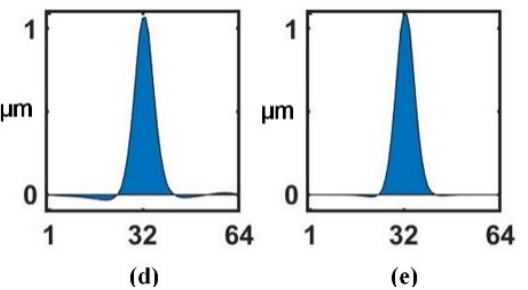

(e)

Figure 6. Cutaway views of the surface shape deformation of (a) $F_{a}(25)$, (b) $F_{\text {measu }}(25)$, (c) $F_{\text {modu1 }}(25)$, (d) $F_{\text {modu2 }}(25)$, and (e) $F_{\text {modu3 }}(25)$.

It could be seen that random fluctuations of the IF filtered by the cut-type filtering $\left(F_{\text {modu } 1}\right)$ are eliminated. The IF filtered by the linear-type filtering $\left(F_{\text {modu } 2}\right)$ has a linear peak and the random fluctuations outside the central area are depressed, while the IF filtered by the Gaussian-type filtering $\left(F_{\text {modu } u}\right)$ has a most concentrated peak and the random fluctuations outside the central area are eliminated. To investigate the difference between $F_{\text {modu } 1}, F_{\text {modu } 2}, F_{\text {modu } u}$ and $F_{\text {measu }}$, the IF deviation characteristics $(\Delta)$ from the ideal IF $F_{a}$ are given by

$$
\left\{\begin{array}{rl}
\Delta_{\text {measu }} & =F_{\text {measu }}-F_{a} \\
\Delta_{\text {modu } 1} & =F_{\text {modu } 1}-F_{a} \\
\Delta_{\text {modu } 2} & =F_{\text {modu } 2}-F_{a} \\
\Delta_{\text {modu } 3} & =F_{\text {modu } 3}-F_{a}
\end{array} .\right.
$$

Here, $\Delta$ is defined as the difference between the IF $F_{c}$ and the ideal IF $F_{a}$. Each IF deviation $\Delta$ consists of $\Delta(i)$ for all the 49 actuators. To evaluate the deviation between the IF $F_{c}$ and the ideal IF $F_{a}$, the RMS values of the IF deviation $\Delta(i)$ are calculated for each actuator. Under ideal measurement conditions, the IF $F_{c}$ equals the ideal IF $F_{a}$, which means the IF deviation $\Delta$ is 0 . Otherwise, the IF $F_{c}$ is not equal to the ideal IF $F_{a}$, and the RMS values of the IF deviation $\Delta(i)$ could be used to evaluate the difference.

As can be seen in Figure 7, for all of the 49 actuators, the RMS values of the IF deviation $\Delta_{\text {measu }}(i)$ range from 0.038 to $0.095 \mu \mathrm{m}$, while those of the $\Delta_{\text {modu1-3 }}(i)$ from 0.011 to $0.060 \mu \mathrm{m}$. For each actuator, the RMS value of the IF deviation $\Delta_{\text {measu }}(i)$ is larger than that of the IF deviation $\Delta_{\text {modu1-3 }}(i)$. The difference is even larger than $50 \%$ for some actuators (e.g., 6th, 16th and 45th actuators). This indicates that the IF deviation between the measured IF $F_{\text {measu }}$ and the ideal IF $F_{a}$ is larger than that between the filtered IFs $F_{\text {modu1-3 }}$ and the ideal IF $F_{a}$. In other words, the deviation between the measured IF and the ideal IF $F_{a}$ brought by the measurement noise $\delta$ could be effectively reduced by using the IFFM. 


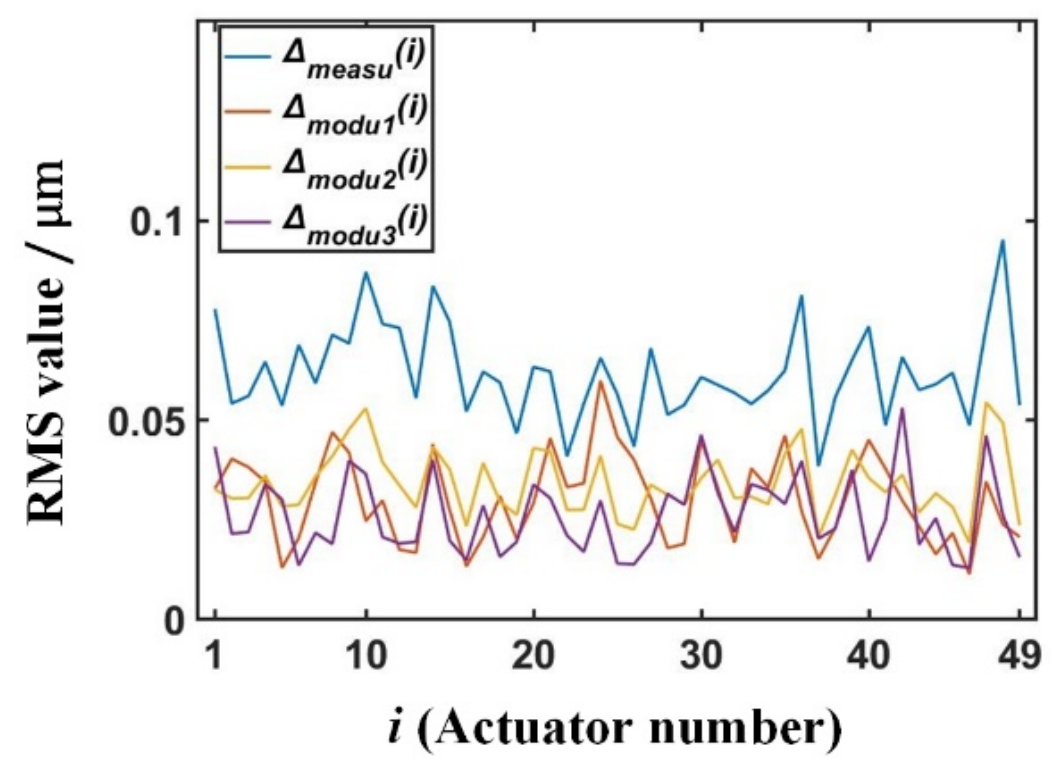

Figure 7. RMS values of the IF deviation $\Delta_{\text {measu }}(i), \Delta_{\text {modu } 1}(i), \Delta_{\operatorname{modu} u}(i)$ and $\Delta_{\text {modu } 3}(i)$ for each actuator.

\section{Simulation}

A simulation is set up to further investigate the improvement by the IFFM on the wavefront correction capability of an $\mathrm{AO}$ system. $F_{\text {measu }}, F_{\text {modu1 } 1}, F_{\text {modu2 }}$ and $F_{\text {modu } 3}$ are set as $F_{c}$ in step one of the CAM to, respectively, calculate the control signal $u$ (Equation (1)), which is then used along with the ideal IF $F_{a}$ to accomplish the wavefront compensation and calculate the residual $r$ in step two. The 3rd to 10th Zernike mode aberrations, as well as an actual wavefront measured in the experiment, are set as the initial wavefront $w$, respectively. The PV and RMS values of the correction residuals of the Zernike mode aberrations are displayed in Figure 8.

PV values of residuals

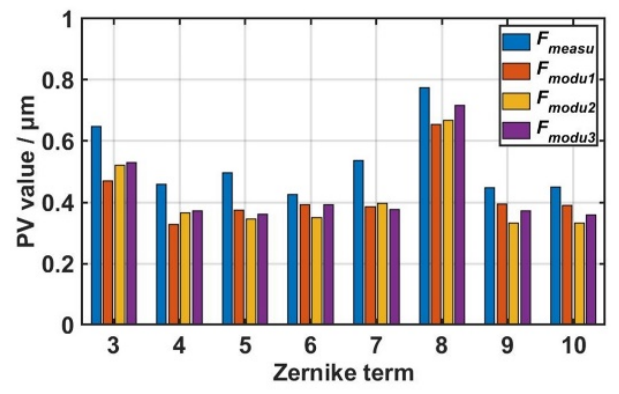

(a)
RMS values of residuals

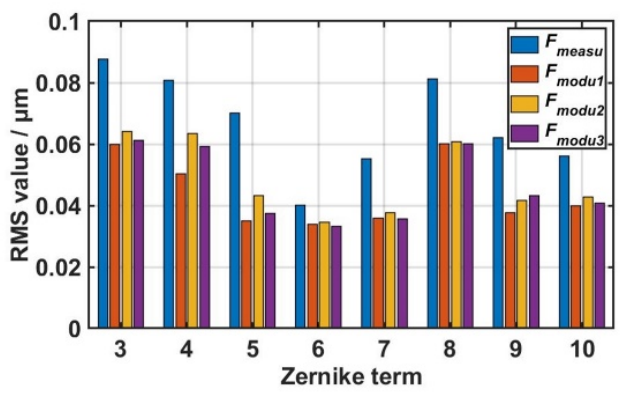

(b)

Figure 8. (a) PV values and (b) RMS values of correction residuals of the 3rd-10th Zernike mode aberrations by using $F_{\text {measu }}, F_{\text {modu } 1}, F_{\text {modu2 } 2}$, and $F_{\text {modu } 3}$. 
As shown in Figure 8, for the 3rd to 10th Zernike mode aberrations, the PV and RMS values of the residual $r$ by using $F_{\text {modu }}$ are, on average, 20\% and 30\% better than those by using $F_{\text {measu }}$, respectively. For instance, for the 6th Zernike mode aberrations, the PV values of the residual $r$ by using $F_{\text {modu1-3 }}(0.393,0.349$ and $0.391 \mu \mathrm{m})$ are $11 \%$ lower on average than those by $F_{\text {measu }}(0.425 \mu \mathrm{m})$, while the RMS values by using $F_{\text {modu1-3 }}(0.034$, 0.035 and $0.033 \mu \mathrm{m})$ are $15 \%$ lower on average than those by using $F_{\text {measu }}(0.040 \mu \mathrm{m})$. As for the 7th Zernike mode aberrations, the PV values of the residual $r$ by using $F_{\text {modu1-3 }}(0.386$, 0.397 and $0.378 \mu \mathrm{m})$ are $28 \%$ lower on average than those by using $F_{\text {measu }}(0.538 \mu \mathrm{m})$, while the RMS values by using $F_{\text {modu1-3 }}(0.036,0.038$ and $0.036 \mu \mathrm{m})$ are $33 \%$ lower on average than those by $F_{\text {measu }}(0.055 \mu \mathrm{m})$. It should be noted that the PV and RMS values of the residual $r$ by using $F_{\text {modu } 1}, F_{\text {modu } 2}$ and $F_{\text {modu } 3}$ have little difference. As shown in Figure 8 , the maximum difference between the PV values of the residual $r$ by using the three filtered IFs is $16 \%$ at the 9 th Zernike mode aberration correction, while the maximum difference between the RMS values is $50 \%$ at the 5 th Zernike mode aberration correction. From Figure $8 \mathrm{a}$ it could be seen that $F_{m o d u 1}$ achieves the lowest PV value of correction residuals for the 3rd, 4th and 8th Zernike mode aberrations, $F_{\text {modu2 }}$ achieves the lowest PV value of correction residuals for the 5 th, 6 th, 9 th and 10 th Zernike mode aberrations, while $F_{\text {modu } 3}$ performs the best for the 7 th Zernike mode aberrations. From Figure $8 \mathrm{~b}$ it could be seen that in terms of the RMS values of correction residuals, $F_{\text {modu } 1}$ performs the best for the 3 rd to 5 th and the 8 th to 10 th Zernike mode aberrations, while $F_{\text {modu } 3}$ performs the best for the 6th and 7th Zernike mode aberrations. It should be noticed that for the 5th and 7th Zernike mode aberrations, the difference of RMS values of correction residuals by using $F_{\text {modu } 1}$ and $F_{\text {modu } 3}$ are less than $3 \%$ and $1 \%$, respectively. The result indicates that for the correction of the 3 rd to 10 th Zernike mode aberrations, $F_{\text {modu } 1-3}$ perform similarly and better than $F_{\text {measu }}$.

To further investigate the effect of the IFFM on the wavefront correction capability in simulation, in addition to the Zernike mode aberrations, an actual wavefront measured in the experiment is taken as the initial wavefront $w$ (Figure 9a). Figure 9b shows that the initial wavefront mainly contains the 13th, 22nd and 24th Zernike mode aberrations. Illustrated in Figure $9 \mathrm{c}-\mathrm{g}$ are the correction results by using $F_{\text {measu }}, F_{\text {modu1 }}, F_{\text {modu2 }}$ and $F_{\text {modu3 }}$, respectively. As shown in Figure $9 \mathrm{~d}$, the residual $r$ by using $F_{\text {measu }}$ has the largest PV value of $0.671 \mu \mathrm{m}$ and the largest RMS value of $0.094 \mu \mathrm{m}$. The PV values of the residual $r$ by using $F_{\text {modu } 1}(0.475 \mu \mathrm{m}), F_{\text {modu } 2}(0.518 \mu \mathrm{m})$ and $F_{\text {modu } 3}(0.482 \mu \mathrm{m})$ are $29 \%, 23 \%$ and $28 \%$ lower than those by $F_{\text {measu }}$, while the RMS values by using $F_{\text {modu } 1}(0.064 \mu \mathrm{m}), F_{\text {modu } 2}$ $(0.078 \mu \mathrm{m})$ and $F_{\text {modu } 3}(0.070 \mu \mathrm{m})$ are $32 \%, 18 \%$ and $25 \%$ lower than those by $F_{\text {measu }}$. This difference indicates that the correction results by using $F_{\text {modu1 } 1}, F_{\text {modu } 2}$ and $F_{\text {modu } 3}$ are better than those by using $F_{\text {measu }}$. Furthermore, the surface shape of the residual by using $F_{\text {modu1-3 }}$ (Figure 9e-g) are smoother than that by using $F_{\text {measu }}$ (Figure 9c). Among the three filtered IFs, $F_{\text {modu } 1}$ achieves the lowest PV and RMS values of correction residuals. $F_{\text {modu2-3 }}$ perform worse but still better than $F_{\text {measu }}$. In conclusion, the wavefront correction capability of the AO system could be effectively improved by using the IFFM. 


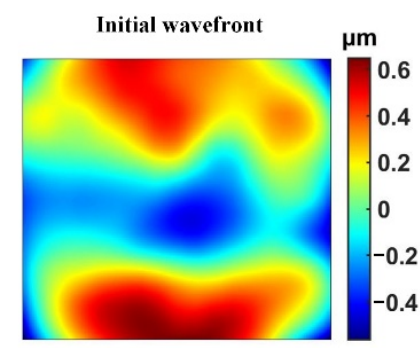

(a)

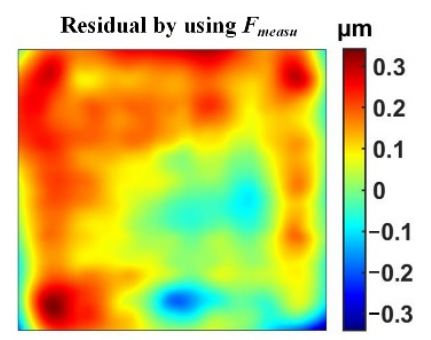

(c)

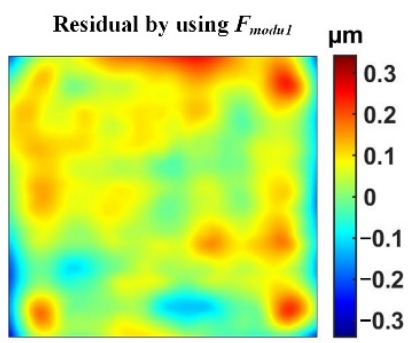

(e)

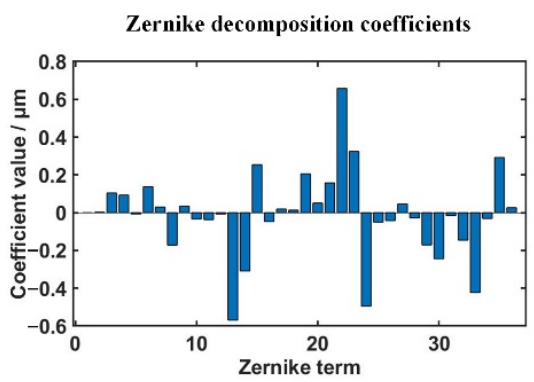

(b)

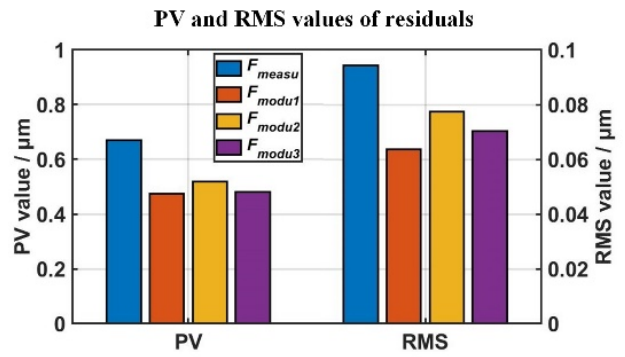

(d)

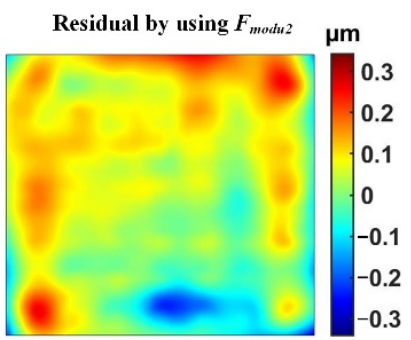

(f)

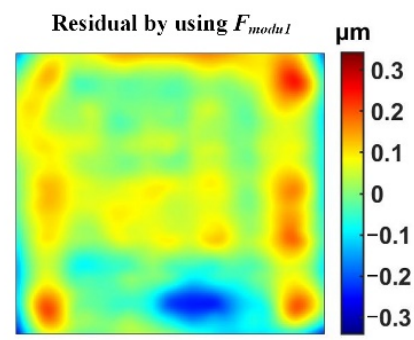

(g)

Figure 9. Correction results of a measured wavefront by using $F_{\text {measu }}, F_{\text {modu1 } 1}, F_{\text {modu2 }}$, and $F_{\text {modu } 3}$. (a) Initial wavefront. (b) Zernike decomposition coefficients of the initial wavefront. (c) Residual by using $F_{\text {measu }}$. (d) PV and RMS values of the residual. (e) Residual by using $F_{\text {modu1 }}$. (f) Residual by using $F_{\text {modu2 }}$. (g) Residual by using $F_{\text {modu } 3}$.

\section{Experiment}

An experiment is conducted to investigate the improvement of the wavefront correction capability by the IFFM under practical conditions. The configuration of the experimental setup is displayed in Figure 10.

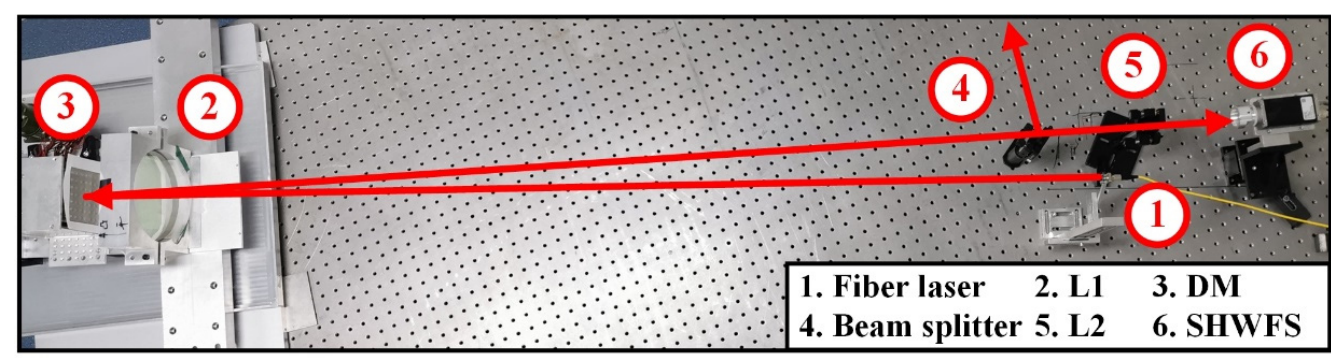

Figure 10. Configuration of the experimental setup.

The laser beam emitted from a fiber laser (Zweda Technology, $1053 \mathrm{~nm}, 0.14$ numerical aperture fiber) first travels through lens L1 (1.5 m focal length) and then is reflected by the lab-manufactured DM $(84 \times 84 \mathrm{~mm}$ size, 49 square-distribution actuators $)$ to a beam splitter where it is divided into two beams. One beam is reflected by the splitter into the rest of the optical path, and the other travels through the splitter and is collimated by lens L2 (100 $\mathrm{mm}$ focal length). The collimated beam enters into the SHWFS and its wavefront 
aberration is measured. The SHWFS consists of a micro-lens array $(20 \times 20$ sub-apertures, $300 \mu \mathrm{m}$ sub-aperture interval, $6 \times 6 \mathrm{~mm}$ size) and a charge-coupled device (Basler's piA1000-48gm, $1004 \times 1004$ pixels, $7.4 \times 7.4 \mu \mathrm{m}$ pixel size and $7.4 \times 7.4 \mathrm{~mm}$ sensor size). A personal computer (PC) is employed for data processing and control signal calculation, and a high-voltage driver is employed to apply the control signal to the DM.

The wavefront correction process of the AO system in the experiment includes four steps as illustrated in Figure 11: the IF measurement, the IF filtering, the wavefront measurement and the closed-loop correction. The IF of the DM is measured by using the SHWFS and the obtained data are transmitted to the PC. The IFFM is operated to modulate the measured IF $F_{\text {measu }}$ into three filtered IFs $\left(F_{\text {modu } 1}, F_{\text {modu } 2}\right.$ and $\left.F_{\text {modu } 3}\right)$. The initial wavefront $w$ of the laser beam is measured by the SHWFS. The control signal $u$ is calculated and applied on the DM to generate the compensating wavefront. To compare the wavefront correction capability between the measured IF $F_{\text {meas }}$ and the filtered IFs $\left(F_{\text {modu } 1}, F_{\text {modu } 2}\right.$ and $\left.F_{\text {modu } 3}\right)$, in the experiment, the wavefront correction is carried out both without and with the IF filtering, which is listed as filtering off/on in Figure 11.

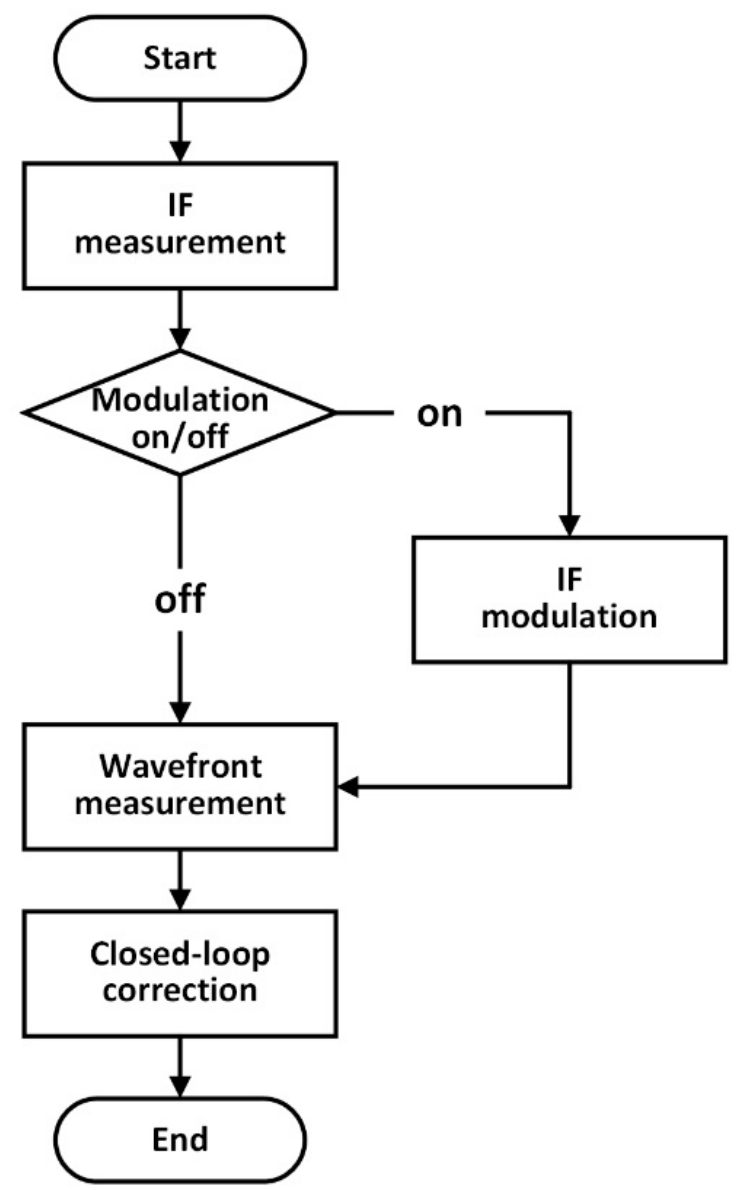

Figure 11. Wavefront correction process of the AO system.

The IF filtering results are shown in Figure 12. Note that unlike the simulation, in the experiment only the actual IF $F_{\text {measu }}$ could be measured while the ideal IF $F_{a}$ of the $\mathrm{DM}$ and the measurement noise $\delta$ could not be obtained. According to Figure 12, the measurement noise exists and the measured IF $F_{\text {measu }}(25)$ has random fluctuations on the whole surface shape. After filtering by using the IFFM, the measurement noises are depressed in $F_{\text {modu } 1}(25), F_{\text {modu2 }}(25)$ and $F_{\text {modu }}(25)$. Based on the measured IF and the filtered IF, the wavefront correction is carried out and the results are illustrated in Figure 13. 


$$
F_{\text {measu }}(\mathbf{2 5 )}
$$

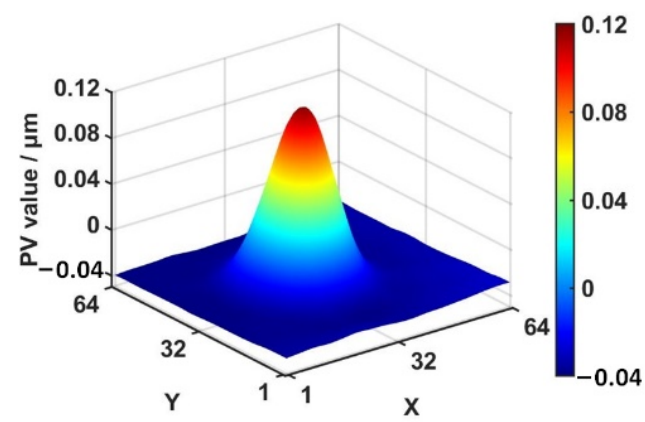

(a)

$$
F_{\text {modu2 } 2}(25)
$$

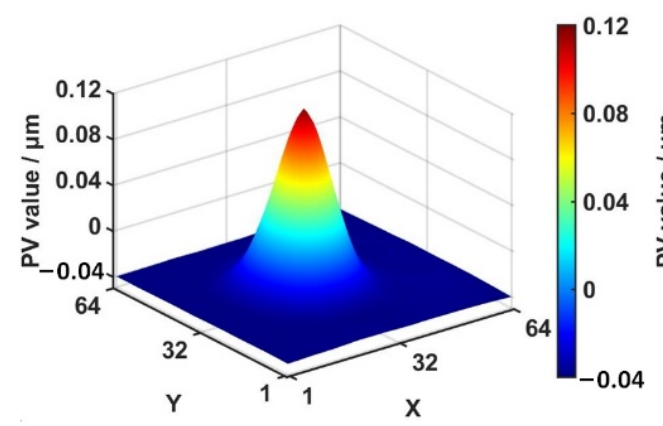

(c)

\section{$F_{\text {modul }}(25)$}

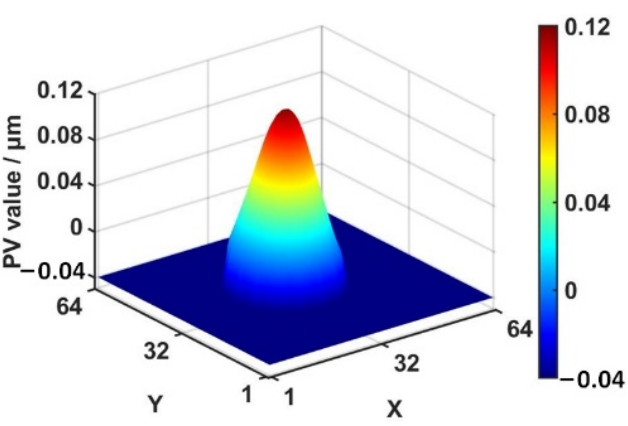

(b)

$F_{\text {modus }}(25)$

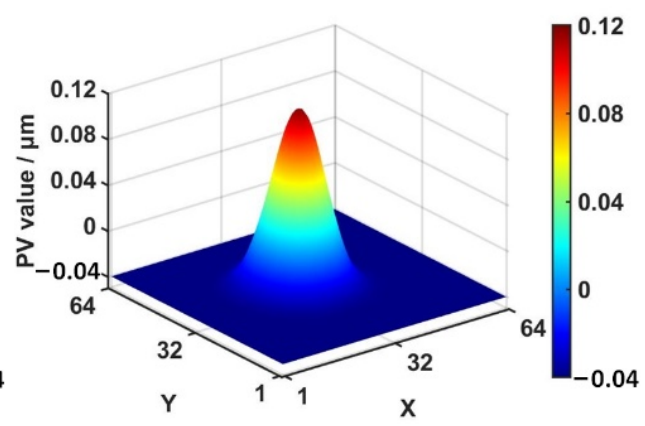

(d)

Figure 12. From the measured IF (a) $F_{\text {measu }}$, the filtered IFs (b) $F_{\text {modu1 }}$, (c) $F_{\text {modu2 }},\left(\right.$ d) $F_{\text {modu3 }}$ for the 25th actuator are achieved.

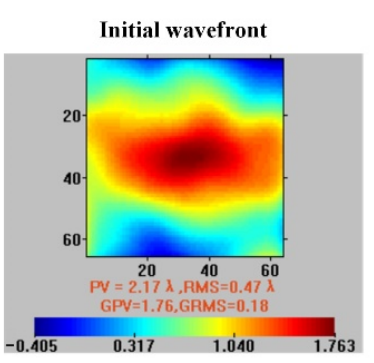

(a)

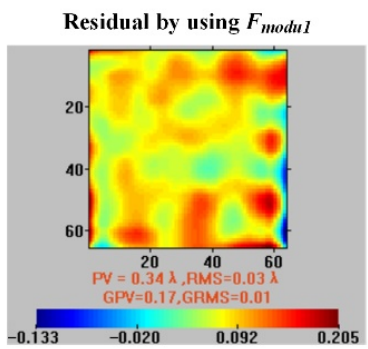

(d)

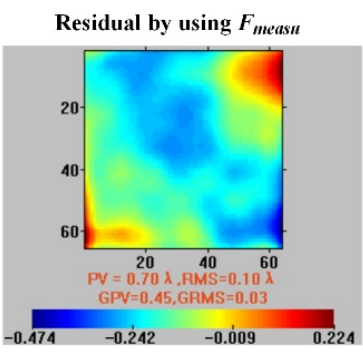

(b)

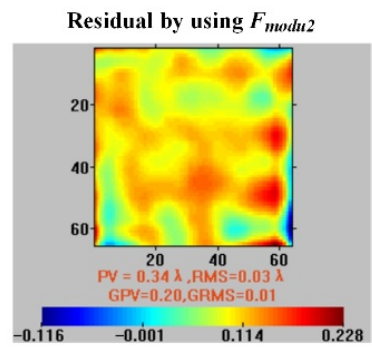

(e)

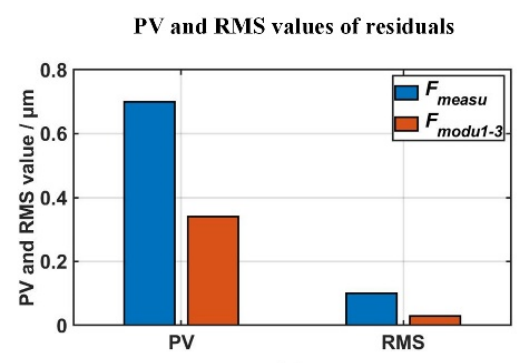

(c)

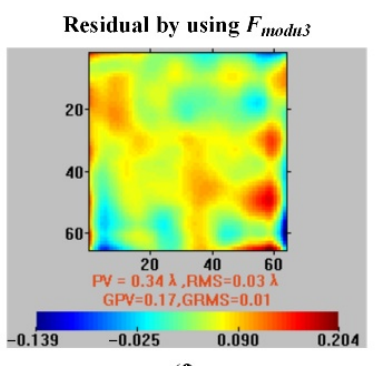

(f)

Figure 13. Correction results by using the measured IF $F_{\text {measu }}$ and three filtered IFs $F_{\text {modu1-3 }}$. (a) Initial wavefront. (b) Residual by using $F_{\text {measu }}$. (c) PV and RMS values of residuals. (d) Residual by using $F_{\text {modu1 }}$. (e) Residual by using $F_{\text {modu2 }}$. (f) Residual by using $F_{\text {modu3 }}$. 
As shown in Figure 13, the initial wavefront has the PV value of $2.17 \lambda(\lambda=1053 \mathrm{~nm})$ and the RMS value of $0.47 \lambda$. After correction by $F_{\text {measu }}$, the initial aberration is depressed and the residual wavefront $r$ has the PV value of $0.70 \lambda$ and the RMS value of $0.10 \lambda$. It could be seen from Figure 13b that there still exists uncorrected aberrations (e.g., a distinct local convex on the right top of the residual wavefront) in the residual wavefront corrected by $F_{\text {measu }}$. The possible causes of the uncorrected aberrations are the measurement noise, the correction algorithm, the system structure, the DM structure and the linearity error of the correction. Compared with $F_{\text {measu }}$, the correction results by using $F_{\text {modu } 1}, F_{\text {modu } 2}$ and $F_{\text {modu } 3}$ are improved with a $51 \%$ reduction in PV value and a $97 \%$ reduction in RMS value, while no distinct uncorrected aberrations occur in the residual wavefronts. Thus, the residual wavefronts corrected by the filtered three IFs are much smoother than those corrected by the measured IF, as can be seen in Figure 13. From Figure 13d-f it could be seen that the three residuals differ from each other in surface shape distribution. In the upper-right area of the residuals, the largest local wavefront variation comes from the residual corrected by $F_{\text {modu } 1}$, while the smallest comes from $F_{\text {modu } 3}$. In the central area, the local wavefront variation of the residuals corrected by $F_{\text {modu } 1}$ and $F_{\text {modu } 3}$ are both smaller than that by $F_{\text {modu2 }}$. Nevertheless, it could also be seen that the PV and RMS values of the closed-loop residuals corrected by the three filtered IFs $F_{\text {modu1-3 }}$ are very close, with differences both less than $0.1 \lambda$. In terms of the closed-loop correction capability, the three filtered IFs are close to each other. In conclusion, experimental results indicate that the wavefront correction results of $F_{\text {modu } 1}, F_{\text {modu } 2}$ and $F_{\text {modu } 3}$ are better than $F_{\text {measu }}$, and that the IFFM could effectively improve the wavefront correction capability of the practical AO system.

\section{Discussion}

In this paper, we propose the IFFM to improve the closed-loop correction capability of an $\mathrm{AO}$ system. In a practical wavefront sensor $\mathrm{AO}$ system, the measured IF and wavefront are used to calculate the control signals of the DM. As we know, the measurement is nonideal and the measurement noises always exist, which would result in the difference between the measured IF and the ideal IF. This difference finally leads to the failure to calculate the optimal control signal during each iteration of the closed-loop correction, and thus the correction ability of the $\mathrm{AO}$ system is degraded. The proposed IFFM depresses the measurement noises by filtering the measured IF into a new IF for the calculation of the control signals. With the filtered IF, the control signal of the closed-loop correction could converge to the optimal one, which improves the correction capability of the AO system. Compared with the measurement accuracy improvement methods proposed in [26-28], which require multiple IF measurements in the closed-loop correction process, the IFFM requires only a single IF measurement in the precorrection process, making the overall correction process more controllable. Meanwhile, the high precision IF measurement and fitting methods proposed in [29-31] could also help improve the closed-loop correction ability, while these methods are implemented with additional high precision surface measuring equipment. Since the IFFM is operated on a software platform without the need of additional measuring equipment, compared with these methods proposed in [29-31], the IFFM has better applicability and flexibility as well as practicality.

Nonetheless, the IFFM is inapplicable to the wavefront sensorless AO system where neither IF measurement nor wavefront measurement is taken for closed-loop correction. For example, the closed-loop correction mentioned in $[19,20]$ takes the far-field beam quality as the evaluation factor without the IF measurement.

It should also be noticed that the IFFM is implemented on the wavefront controller during the precorrection process. For an AO system, the implementation of the IFFM requires no additional hardware and does not affect the correction speed. At the same time, the calculation of the IFFM is relatively simple and does not need to occupy a lot of computing resources of the wavefront controller. Finally, our method provides an approach with good applicability and practicability to improve the closed-loop correction capability 
of an $\mathrm{AO}$ system in the case of measurement noise. As can be seen from the simulation and experimental results, all the three filtered IFs we present could be adopted to reduce the measurement noise effectively. The difference between the three methods is small and could be chosen according to experiment conditions and needs.

\section{Conclusions}

This paper presents the IFFM to depress the negative effect of the IF measurement noise on the wavefront correction capability of the AO system in laser systems. The CAM is proposed to investigate the effect of the IF measurement noise and the improvement of the IFFM on the correction ability. Three types of filtering methods are devised in the IFFM to obtain the filtered IF from the measured IF. In the simulation, the Zernike mode aberrations and an actual measured wavefront are taken as the correction object. Simulation results reveal that the occurrence of measurement noise will degrade the wavefront correction capability and the filtered IF has better correction capability than the measured IF, as the filtered IFs have characteristics of less random fluctuations and are closer to the ideal IF. An experiment is carried out to investigate the improvement of the IFFM on the wavefront correction capability. Both simulation and experimental results indicate that the IFFM could depress the negative effect of the IF measurement noise and improve the wavefront correction capability effectively. Additionally, the IFFM does not require external hardware and affects the correction speed of the AO system.

Author Contributions: Conceptualization, L.H.; Data curation, Y.Z.; Formal analysis, Y.Z.; Funding acquisition, L.H.; Investigation, Y.Z.; Methodology, Y.Z.; Project administration, L.H.; Resources, M.L. and L.H.; Software, Y.Z. and S.L.; Supervision, L.H.; Validation, D.W. and Q.X.; Visualization, Y.Z.; Writing-original draft, Y.Z.; Writing-review \& editing, Y.Z. and L.H. All authors have read and agreed to the published version of the manuscript.

Funding: This research was funded by Science and Technology on Plasma Physics Laboratory, China Academy of Engineering Physics, grant number 6142A04180304 and National Natural Science Foundation of China, grant number 61775112.

Data Availability Statement: The data presented in this study are available on request from the corresponding author.

Acknowledgments: Than authors are grateful to other colleagues for their help during the period of experimental measurement.

Conflicts of Interest: The authors declare no conflict of interest.

\section{References}

1. Zhang, X.; Arcidiacono, C.; Conrad, A.R.; Herbst, T.M.; Gaessler, W.; Bertram, T.; Ragazzoni, T.; Schreber, L.; Diolaiti, E.; Kuerster, M.; et al. Calibrating the interaction matrix for the LINC-NIRVANA high layer wavefront sensor. Opt. Express 2012, 20, 8078-8092. [CrossRef] [PubMed]

2. Lardiere, O.; Conan, R.; Bradley, C.; Jackson, K.; Herriot, G. A laser guide star wavefront sensor bench demonstrator for TMT. Opt. Express 2008, 16, 5527-5543. [CrossRef] [PubMed]

3. Baranec, C.; Lloyd -Hart, M.; Milton, N.M.; Stalcup, T.; Snyder, M.; Vaitheeswaran, V.; McCarthy, D.; Angel, R. Astronomical imaging using ground-layer adaptive optics. Proc. SPIE 2007, 6691, 66910N. [CrossRef]

4. Van Dam, M.A.; Bouchez, A.H.; Le Mignant, D.; Johansson, E.M.; Wizinowich, P.L.; Campbell, R.D.; Chin, J.C.Y.; Hartman, S.K.; Lafon, R.E.; Stomski, J.P.J.; et al. The W. M. Keck Observatory Laser Guide Star Adaptive Optics System: Performance Characterization. Publ. Astron. Soc. Pac. 2006, 118, 310-318. [CrossRef]

5. $\quad$ Esposito, S.; Riccardi, A.; Fini, L.; Puglisi, A.T.; Pinna, E.; Xompero, M.; Briguglio, R.; Quirós-Pacheco, F.; Stefanini, P.; Guerra, J.C.; et al. First light AO (FLAO) system for LBT: Final integration, acceptance test in Europe, and preliminary on-sky commissioning results. Proc. SPIE 2010, 7736, 773609. [CrossRef]

6. Martinache, F.; Guyon, O.; Clergeon, C.; Garrel, V.; Blain, C. The Subaru coronagraphic extreme AO project: First observations. Proc. SPIE 2012, 8447, 84471Y. [CrossRef]

7. Neichel, B.; Rigaut, F.; Vidal, F.; van Dam, M.A.; Garrel, V.; Carrasco, E.R.; Pessev, P.; Winge, C.; Boccas, M.; d'Orgeville, C.; et al. Gemini multiconjugate adaptive optics system review-II. Commissioning, operation and overall performance. Mon. Not. R. Astron. Soc. 2014, 440, 1002-1019. [CrossRef] 
8. Sauvage, J.; Fusco, T.; Petit, C.; Costille, A.; Mouillet, D.; Beuzit, J.; Dohlen, K.; Kasper, M.; Suarez, M.; Soenke, C.; et al. SAXO: The extreme adaptive optics system ofSPHERE (I) system overview and global laboratory performance. J. Astron. Telesc. Instrum. Syst. 2016, 2, 025003. [CrossRef]

9. Rahman, S.A.; Booth, M.J. Direct wavefront sensing in adaptive optical microscopy using backscattered light. Appl. Opt. 2013, 52, 5523-5532. [CrossRef]

10. Bonora, S.; Jian, Y.F.; Zhang, P.F.; Zam, A.; Pugh, E.N.; Zawadzki, R.J.; Sarunic, M.V. Wavefront correction and high-resolution in vivo OCT imaging with an objective integrated multi-actuator adaptive lens. Opt. Express 2015, 23, 21931-21941. [CrossRef]

11. Sahu, P.; Mazumder, N. Advances in adaptive optics-based two-photon fluorescence microscopy for brain imaging. Lasers Med. Sci. 2020, 35, 317-328. [CrossRef] [PubMed]

12. Zacharias, R.A.; Beer, N.R.; Bliss, E.S.; Burkhar, S.C.; Cohen, S.J.; Sutton, S.B.; Van Atta, R.L.; Winters, S.E.; Salmon, J.T.; Stolz, C.J.; et al. National Ignition Facility alignment and wavefront control. Proc. SPIE 2004, 5341, 168-179. [CrossRef]

13. Sacks, R.; Auerbach, J.; Bliss, E.; Henesian, M.; Lawson, J.; Manes, K.; Renard, P.; Salmon, T.; Trenholme, J.; Williams, W.; et al. Application of adaptive optics for controlling the NIF laser performance and spot size. Proc. SPIE 1999, 3492, 344-354. [CrossRef]

14. Van Wonterghem, B.M.; Murray, J.R.; Campbell, J.H.; Speck, D.R.; Barker, C.E.; Smith, I.C.; Browning, D.F.; Behrendt, W.C. Performance of a prototype for a large-aperture multipass Nd:glass laser for inertial confinement fusion. Appl. Opt. 1997, 36, 4932-4953. [CrossRef]

15. Hammer, D.X.; Ferguson, R.D.; Bigelow, C.E.; Iftimia, N.V.; Ustun, T.E.; Burns, S.A. Adaptive optics scanning laser ophthalmoscope for stabilized retinal imaging. Opt. Express 2006, 14, 3354-3367. [CrossRef]

16. Fernandez, E.J.; Vabre, L.; Hermann, B.; Unterhuber, A.; Povazay, B.; Drexler, W. Adaptive optics with a magnetic deformable mirror: Applications in the human eye. Opt. Express 2006, 14, 8900-8917. [CrossRef]

17. Marcos, S.; Werner, J.S.; Burns, S.A.; Merigan, W.H.; Artal, P.; Atchison, D.A.; Hampson, K.M.; Legras, R.; Lundstrom, L.; Yoon, G.; et al. Vision science and adaptive optics, the state of the field. Vision Res. 2017, 132, 3-33. [CrossRef]

18. Goodno, G.D.; Komine, H.; McNaught, S.J.; Weiss, S.B.; Redmond, S.; Long, W.; Simpson, R.; Cheung, E.C.; Howland, D.; Epp, P.; et al. Coherent combination of high-power, zigzag slab lasers. Opt. Lett. 2006, 31, 1247-1249. [CrossRef]

19. Sun, L.C.; Guo, Y.D.; Shao, C.F.; Li, Y.; Zheng, Y.M.; Sun, C.; Wang, X.J.; Huang, L. 10.8 kW, 2.6 times diffraction limited laser based on a continuous wave Nd:YAG oscillator and an extra-cavity adaptive optics system. Opt. Lett. 2018, 43, 4160-4163. [CrossRef]

20. Xu, L.; Wu, Y.C.; Du, Y.L.; Wang, D.; An, X.C.; Li, M.; Zhou, T.J.; Shang, J.L.; Wang, J.T.; Liu, Z.W.; et al. High brightness laser based on Yb:YAG MOPA chain and adaptive optics system at room temperature. Opt. Express 2018, 26, 14592-14600. [CrossRef]

21. Brousseau, D.; Borra, E.F.; Thibault, S. Wavefront correction with a 37-actuator ferrofluid deformable mirror. Opt. Express 2007, 15, 18190-18199. [CrossRef]

22. Bonora, S. Distributed actuators deformable mirror for adaptive optics. Opt. Commun. 2011, 284, 3467-3473. [CrossRef]

23. Gonzalez-Nunez, H.; Bechet, C.; Ayancan, B.; Neichel, B.; Guesalaga, A. Effect of the influence function of deformable mirrors on laser beam shaping. Appl. Opt. 2017, 56, 1637-1646. [CrossRef]

24. Fernandez, E.J.; Artal, P. Membrane deformable mirror for adaptive optics: Performance limits in visual optics. Opt. Express 2003, 11, 1056-1069. [CrossRef] [PubMed]

25. Mansell, J.; Jameson, J.; Henderson, B. Advanced deformable mirrors for high-power lasers. Proc. SPIE 2014, $9083,90830 \mathrm{~S}$. [CrossRef]

26. Zou, W.Y.; Burns, S.A. High-accuracy wavefront control for retinal imaging with Adaptive-Influence-Matrix Adaptive Optics. Opt. Express 2009, 17, 20167-20177. [CrossRef] [PubMed]

27. Huang, L.; Ma, X.K.; Bian, Q.; Li, T.H.; Zhou, C.L.; Gong, M.L. High-precision system identification method for a deformable mirror in wavefront control. Appl. Opt. 2015, 54, 4313-4317. [CrossRef]

28. Ren, Z.L.; Liu, J.; Zhang, Z.T.; Chen, Z.T.; Liang, Y.H. High-resolution wavefront reconstruction using multiframe Shack-Hartmann wavefront sensor measurements. Opt. Eng. 2020, 59, 113102. [CrossRef]

29. Zheng, Y.M.; Sun, C.; Dai, W.J.; Zeng, F.; Xue, Q.; Wang, D.E.; Zhao, W.C.; Huang, L. High precision wavefront correction using an influence function optimization method based on a hybrid adaptive optics system. Opt. Express 2019, 27, 34937-34951. [CrossRef]

30. Huang, L.H.; Rao, C.H.; Jiang, W.H. Modified Gaussian influence function of deformable mirror actuators. Opt. Express 2008, 16, 108-114. [CrossRef]

31. Roopashree, M.B.; Vyas, A.; Prasad, B.R. Influence Function Measurement of Continuous Membrane Deformable Mirror Actuators Using Shack Hartmann Sensor. AIP Conf. Proc. 2011, 1391, 453-455. [CrossRef]

32. Rosen, S.; Eldert, C. Least-Squares Method for Optical Correction. J. Opt. Soc. Am. 1954, 44, 250-252. [CrossRef]

33. Claflin, E.S.; Bareket, N. Configuring an electrostatic membrane mirror by least-squares fitting with analytically derived influence functions. J. Opt. Soc. Am. 1986, 3, 1833-1839. [CrossRef]

34. Feng, Z.X.; Huang, L. Over compensation algorithm for laser beam shaping using a deformable freeform mirror. Optik 2019, 198, 163250. [CrossRef]

35. Tabatabaian, M. COMSOL for Engineers; Mercury Learning \& Information: Herndon, VA, USA, 2014.

36. Higham, D.J.; Higham, N.J. MATLAB Guide, 3rd ed.; Siam: Philadelphia, PA, USA, 2016.

37. Wang, J.Y.; Silva, D.E. Wave-front interpretation with Zernike polynomials. Appl. Opt. 1980, 19, 1510-1518. [CrossRef] [PubMed]

38. Alda, J.; Boreman, G.D. Zernike-based matrix model of deformable mirrors: Optimization of aperture size. Appl. Opt. 1993, 32, 2431-2438. [CrossRef] 